Abstract

\title{
Functionals of Harmonics Functions ${ }^{\dagger}$
}

\author{
Niurka R. Quintero ${ }^{1,2, *}$, José A. Cuesta ${ }^{3,4}$ and Renato Alvarez-Nodarse 5
}

1 Departamento de Física Aplicada I, E.P.S., Universidad de Sevilla, Virgen de África 7, 41011 Sevilla, Spain Instituto Carlos I de Física Teórica y Computacional, Universidad de Granada, 18071 Granada, Spain

3 Grupo Interdisciplinar de Sistemas Complejos (GISC), Departamento de Matemáticas, Universidad Carlos III de Madrid, Avda. de la Universidad 30, 28911 Leganes, Spain; cuesta.jose.a@gmail.com

4 Instituto de Biocomputación y Física de Sistemas Complejos (BIFI), Universidad de Zaragoza, 50009 Zaragoza, Spain

5 Departamento de Análisis Matemático, Universidad de Sevilla, Apdo 1160, 41080 Sevilla, Spain; ran@us.es

* Correspondence: nrquintero@gmail.com

+ Presented at Symmetry 2017-The First International Conference on Symmetry, Barcelona, Spain, 16-18 October 2017.

Published: 9 January 2018

Let $\mathcal{C}_{T}^{s}$, with $T>0$, be the set of continuous, $T$-periodic functions $\mathbf{f}: \mathbb{R} \rightarrow \mathbb{R}^{s}$, and let $\Gamma: \mathcal{C}_{T}^{s} \rightarrow \mathbb{R}$ be a real functional on $\mathcal{C}_{T}^{s}$. If $\Gamma$ is $n$ times Fréchet differentiable on $\mathcal{C}_{T}^{s}$, then it has an $n$-th order Taylor expansion around $\mathbf{0}$ (see e.g., [1]). Such a Taylor expansion can be obtained as the $n$-th order truncation of the series

$$
\begin{aligned}
\Gamma[\mathbf{f}]= & \sum_{n_{1}=0}^{\infty} \cdots \sum_{n_{s}=0}^{\infty}\left\langle c_{\mathbf{n}}\left(t_{11}, \ldots, t_{1 n_{1}}, \ldots, t_{s 1}, \ldots, t_{s n_{s}}\right)\right. \\
& \left.\times f_{1}\left(t_{11}\right) \cdots f_{1}\left(t_{1 n_{1}}\right) \cdots f_{s}\left(t_{s 1}\right) \cdots f_{s}\left(t_{s n_{s}}\right)\right\rangle,
\end{aligned}
$$

where $\mathbf{n}=\left(n_{1}, \ldots, n_{s}\right)$ and we have introduced the notation

$$
\left\langle\Omega\left(t_{1}, \ldots, t_{r}\right)\right\rangle=\frac{1}{T^{r}} \int_{0}^{T} d t_{1} \cdots \int_{0}^{T} d t_{r} \Omega\left(t_{1}, \ldots, t_{r}\right) .
$$

The kernels $c_{n_{1}, \ldots, n_{s}}\left(t_{11}, \ldots, t_{s n_{s}}\right)$ are all real, $T$-periodic, and symmetric in all their arguments. In this contribution we will prove the following theorem.

Theorem 1. Let $\Gamma$ be a functional with Taylor series (1), and take

$$
\mathbf{f}(t)=\left(\epsilon_{1} \cos \left(q_{1} \omega t+\phi_{1}\right), \ldots, \epsilon_{s} \cos \left(q_{s} \omega t+\phi_{s}\right)\right),
$$

where $\mathbf{q} \equiv\left(q_{1}, \ldots, q_{s}\right) \in \mathbb{N}^{s}$ is such that $\operatorname{gcd}\left(q_{1}, \ldots, q_{s}\right)=1$ and $\omega=2 \pi / T$. Then,

$$
\Gamma[\mathbf{f}]=C_{\mathbf{0}}(\boldsymbol{\epsilon})+\sum_{\mathbf{x} \in S_{+}} \epsilon_{1}^{\left|x_{1}\right|} \cdots \epsilon_{S}^{\left|x_{s}\right|} C_{\mathbf{x}}(\boldsymbol{\epsilon}) \cos \left(\mathbf{x} \cdot \boldsymbol{\phi}+\theta_{\mathbf{x}}(\boldsymbol{\epsilon})\right),
$$

where, $\boldsymbol{\phi} \equiv\left(\phi_{1}, \ldots, \phi_{s}\right), \boldsymbol{\epsilon} \equiv\left(\epsilon_{1}, \ldots, \epsilon_{S}\right)$, and functions $C_{\mathbf{x}}(\boldsymbol{\epsilon})$ and $\theta_{\mathbf{x}}(\boldsymbol{\epsilon})$ do not depend on $\boldsymbol{\phi}$ and are even in each $\epsilon_{i}, i=1, \ldots, s$, for every $\mathbf{x} \in S_{+} . \mathbf{x} \in S_{+}$is the set of vectors $\mathbf{x}$ whose leftmost nonzero component is positive.

In the special case when $\Gamma$ is invariant under time-shift, i.e., $\Gamma[\mathbf{f}(t+\tau)]=\Gamma[\mathbf{f}(t)]$ for all $0<\tau<T$, we recover the results in [2]

$$
\Gamma[\mathbf{f}]=C_{\mathbf{0}}(\boldsymbol{\epsilon})+\sum_{\mathbf{x} \in \mathcal{D}_{+}} \epsilon_{1}^{\left|x_{1}\right|} \cdots \epsilon_{s}^{\left|x_{s}\right|} C_{\mathbf{x}}(\boldsymbol{\epsilon}) \cos \left(\mathbf{x} \cdot \boldsymbol{\phi}+\theta_{\mathbf{x}}(\boldsymbol{\epsilon})\right),
$$


where $\mathcal{D}_{+}$denote the set of nonzero solutions of the Diophantine equation $\mathbf{q} \cdot \mathbf{x}=q_{1} x_{1}+\cdots+q_{s} x_{s}=0$, whose leftmost nonzero component is positive.

Acknowledgments: We acknowledge financial support from the MINECO of Spain through FIS2014-54497-P (N.R.Q.) and MTM2015-65888-C4-1-P (R.A.N.).

\section{References}

1. Wouk, A. Course of Applied Functional Analysis; John Wiley \& Sons: New York, NY, USA, 1979.

2. Cuesta, J.A.; Quintero, N.R.; Alvarez-Nodarse, R. Time-shift invariance determines the functional shape of the current in dissipative rocking ratchets. Phys. Rev. X 2013, 3, 041014.

(C) 2018 by the authors. Licensee MDPI, Basel, Switzerland. This article is an open access article distributed under the terms and conditions of the Creative Commons Attribution (CC BY) license (http:/ / creativecommons.org/licenses/by/4.0/). 